\title{
Editorials
}

\section{Assessment of cancer risk in men and women}

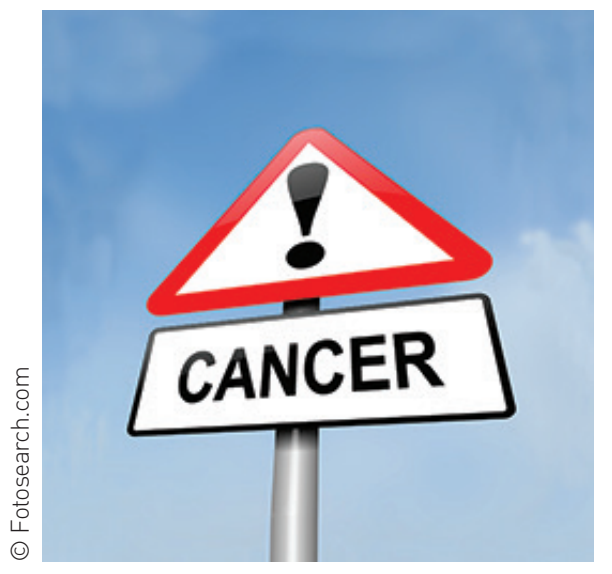

A core element of primary care's role in cancer care is the assessment of cancer risk. Not only do we need to assess the future (or underlying) risk of someone developing cancer, for example based on their family history and lifestyle factors, ${ }^{1}$ we also need to assess the risk that someone has an underlying cancer now. This requires assessment of current symptoms as well as their underlying cancer risk factors to determine whether further investigation is required.

\section{CANCER DIAGNOSIS IN GENERAL PRACTICE}

Diagnosing cancer in general practice is difficult due to the non-specific nature of symptoms associated with common cancers in primary care. ${ }^{2} \mathrm{~A}$ national audit of 2-week wait referrals for suspected cancer reported in this journal confirmed these challenges: only $11 \%$ of fast-track referrals were eventually diagnosed as cancer, and only $43 \%$ of cancers were referred via this route. $^{3}$ These referral routes relied on the National Institute of Health and Clinical Excellence guidelines for suspected cancer which, when published in 2005, had limited evidence derived from primary care on the predictive value of symptoms. Since then the body of evidence has grown dramatically. Hamilton's CAPER studies, and HippisleyCox's QCancer ${ }^{\circledR}$ work, which have used slightly different methodological approaches to examine large general practice datasets, have made important contributions to our understanding of the epidemiology of cancer symptoms in primary care. ${ }^{4-6}$ To date this work has reported the symptomatology of individual cancers.

\section{CANCER DIAGNOSTIC RISK MODELS}

In this issue Hippisley-Cox et al report separate models, for men and women, which predict risks of different cancers according to baseline risk factors, current symptoms, and specific clinical conditions such as anaemia and venous thromboembolism. ${ }^{7.8}$ This has greater clinical relevance because, as one would expect, common symptoms are associated with more than one type of cancer. For example, abdominal pain is associated with eight and nine different cancers in men and women respectively; a change in bowel habit is associated with four cancers in women. These new risk models have significant methodological strengths: they are based on very large, representative general practice datasets (23216 cancers in over 1.2 million women) and the models have been developed and then validated by splitting the data to create two separate cohorts. As the authors have already done for some of their cancer models, ${ }^{9}$ external validation of the models published in this edition are required on a cohort from a different general practice dataset.

\section{TRANSLATING RISK MODELS INTO PRACTICE}

Improving cancer outcomes in England by diagnosing cancer earlier remains a key strategic priority for the Department of Health. ${ }^{10}$ Can the use of cancer risk assessment tools in general practice, such as those based on QCancer or the CAPER studies, lead to earlier cancer diagnosis? The Department of Health has already piloted the CAPER risk charts for lung and colorectal cancer presented on computer mouse-mats, and it is about to commence a wider programme based on electronic implementation of CAPER and QCancer risk models. Internationally, a cluster randomised controlled trial of academic detailing to implement the CAPER risk charts, as part of a complex intervention to reduce diagnostic delay, is in progress in rural Western Australia.

Electronic integration of cancer risk assessment tools within clinical software is a potentially effective strategy to translate them into routine practice. ${ }^{11}$ GPs in the UK are familiar with using electronic cardiovascular risk calculators for primary prevention. These tools allow GPs to combine numerical data, such as lipid levels and blood pressure, to calculate an overall numerical risk. Furthermore, use of these cardiovascular tools receives a Quality and Outcomes Framework incentive payment increasing the likelihood of their use. But will GPs perceive use of cancer risk calculators differently? The assessment of symptoms to reach a provisional diagnosis is a core part of what GPs do, but we do not know yet whether they will use numerical calculators to inform their diagnostic decision making. Doctors may have concerns about using such tools with the patient present and the data presented simply as absolute risks of various cancers. Ideally these risk calculators could be developed further to present risks in more meaningful formats within the clinical software, and linked to guidance on diagnostic and referral pathways.

\section{RISK MODELS AS AN AUDIT TOOL}

There is an alternative approach to use the QCancer models which does not rely on doctors using them within a consultation. The model can be run across the whole practice dataset to identify patients who have recently presented and who fall into a pre-specified level of absolute cancer risk. For example, the top 5\% of risk in women would identify $38.5 \%$ of all the cancers included in the model, with a positive predictive value of $14.2 \%$. This may potentially identify patients who require
"The assessment of symptoms to reach a provisional diagnosis is a core part of what GPs do, but we do not know yet whether they will use numerical calculators to inform their diagnostic decision making. 


\section{"The challenge now is how best to translate these risk models into routine clinical practice."}

follow-up or who could be flagged if they return with ongoing symptoms. Further research is needed to understand the implications of using these risk models in this way, especially in terms of potentially needing to follow-up large numbers of false positives.

General practice now has much better evidence to inform the assessment of patients presenting with symptoms associated with cancer. Cancer risk assessment models have the potential to improve the identification of patients requiring urgent investigation for cancer. The new QCancer models published in this edition offer more precise guidance on which cancer is most likely and therefore which diagnostic pathway is best for a man or woman presenting with a range of common symptoms. The challenge now is how best to translate these risk models into routine clinical practice.

\section{Jon Emery,}

School of Primary, Aboriginal and Rural Health Care, University of Western Australia. General Practice and Primary Health Care Academic Centre, University of Melbourne, Melbourne, Australia.

\section{Competing interests}

The author has declared no competing interests.

\section{Provenance}

Commissioned; not externally peer reviewed.

DOI: 10.3399/bjgp13X660607

\section{REFERENCES}

1. Walter FM, Emery JD. Genetic advances in medicine: has the promise been fulfilled in general practice? Br J Gen Pract 2012; 62(596): 120-121.

2. Rubin G, Vedsted P, Emery J. Improving cancer outcomes: better access to diagnostics in primary care could be critical. Br J Gen Pract 2011; 61(586): 317-318

3. Meechan D, Gildea $C$, Hollingworth $L$, et al. Variation in use of the 2-week referral pathway for suspected cancer: a cross-sectional bjgp12X654551.

4. Shephard EA, Stapley S, Neal RD, et al. Clinical features of bladder cancer in primary care. $\mathrm{Br} J$ Gen Pract 2012; DOI: 10.3399/bjgp12X654560.

5. Hippisley-Cox J, Coupland C. Identifying patients with suspected renal tract cancer in primary care: derivation and validation of an algorithm. $\mathrm{Br}$ J Gen Pract 2012; DOI: 10.3399/bjgp12X636074.

6. Hippisley-Cox J, Coupland C. Identifying patients with suspected pancreatic cancer in primary care: derivation and validation of an algorithm. $\mathrm{Br}$ J Gen Pract 2012; DOI: 10.3399/bjgp12X616355.

7. Hippisley-Cox J, Coupland C. Symptoms and risk factors to identify men with suspected of an algorithm. Br J Gen Pract 2013; DOI: 10.3399/bjgp13X660724.

8. Hippisley-Cox J, Coupland C. Symptoms and risk factors to identify women with suspected cancer in primary care: derivation and validation of an algorithm. Br J Gen Pract 2013; DOI: 10.3399/bjgp13X660733.

9. Collins GS, Altman DG. Identifying patients with undetected colorectal cancer: an independent validation of QCancer (Colorectal). Br J Cancer 2012; 107(2): 260-265.

10. Department of Health. Improving outcomes: a strategy for cancer. London: DoH, 2011.

11. Garg AX, Adhikari NK, McDonald H, et al. Effects of computerized clinical decision support systems on practitioner performance and patient outcomes: a systematic review. JAMA 2005 293(10): 1223-1238. analysis. Br J Gen Pract 2012; DOI: 10.3399/ cancer in primary care: derivation and validation

\section{ADDRESS FOR CORRESPONDENCE}

\section{Jon Emery}

General Practice, School of Primary, Aboriginal and Rural Health Care, University of Western Australia, M706 35 Stirling Highway, Crawley, WA 6009 Australia.

E-mail: Jon.Emeryduwa.edu.au 